UDC 681.513.

DOI: 10.20535/SRIT.2308-8893.2019.1.07

\title{
FUZZY GMDH AND ITS APPLICATION TO FORECASTING FINANCIAL PROCESSES
}

\author{
YURIY ZAYCHENKO, HELEN ZAYCHENKO
}

\begin{abstract}
This paper is devoted to the investigation and application of the fuzzy inductive modeling method known as Group Method of Data Handling (GMDH) in problems of Data Mining, in particularly its application to solving the forecasting tasks in financial sphere. The advantage of the inductive modeling method GMDH is a possibility of constructing an adequate model directly in the process of algorithm run. The generalization of GMDH in case of uncertainty - a new method fuzzy GMDH is described which enables to construct fuzzy models almost automatically. The algorithm of fuzzy GMDH is considered. Fuzzy GMDH with Gaussian and bell-wise membership functions MF are considered and their similarity with triangular MF is shown. Fuzzy GMDH with different partial descriptions orthogonal polynomials of Chebyshev and Fourier are considered. The problem of adaptation of fuzzy models obtained by FGMDH is considered and the corresponding adaptation algorithm is described. The extension and generalization of fuzzy GMDH in case of fuzzy inputs is considered and its properties are analyzed. The experimental investigations of the suggested FGMDH were carried out.
\end{abstract}

Keywords: fuzzy GMDH, membership functions, models adaptation, forecasting.

\section{INTRODUCTION}

One of the most important problems in the sphere of economy and finance is the problem of forecasting economical and financial processes. The distinguishing features of the problem are the following:

- the form of functional dependence is unknown and only model class is determined, for example, polynomial of any degree or Fourier time series;

- short data samples;

- time series $x_{i}(t)$ in general case is non-stationary.

In this case, the application of conventional methods of statistical analysis (e.g. regression analysis) is impossible and it is necessary to utilize methods based on computational intelligence (CI). The Group Method of Data Handling (GMDH) developed by acad. A.G. Ivakhnenko [1] and extended by his colleges [2] belongs to this class. GMDH is a self-organizing method allowing to discover internal hidden laws in the appropriate object area. The advantages of GMDH algorithms are the possibility of constructing optimal models from samples with a small number of observations and unknown relationships among variables. This method does not demand to know the model structure a priori; it is constructed by algorithm itself in the process of its run.

In case if input data are measured with errors which distribution is not normal and is unknown to a decision-maker then it is reasonable to construct fuzzy model using Fuzzy GMDH method. FGMDH was suggested in [3] where main ideas of the method and an algorithm of fuzzy GMDH was presented and investi- 
gated in case when fuzzy coefficients of models have triangular membership functions. For finding optimal parameters of fuzzy model, the LP problem was introduced and solved. Later this method was extended to Gaussian and bell-wise MF [4]. It was shown that the structure of LP model for this problem is the same as for triangular MF. Further FGMDH method was extended to orthogonal polynomials as partial descriptions $[5,6]$.

Problem of adaptation of fuzzy models in FGMDH was considered in [5] and several adaptation methods were suggested and investigated. Numerous experimental investigations of fuzzy GMDH with different partial descriptions and MF were carried out and comparison with classical GMDH was performed [7].

The extension of FGMDH and its generalization in the case when input data is fuzzy were considered in [8]. The math model for determination of fuzzy coefficients was constructed and general FGMDH algorithm for fuzzy inputs was developed and investigated.

The goal of this paper is to present a review of the main results in the field of developing the fuzzy GMDH and experimental results of its applications to the forecasting in financial sphere.

\section{OTHER FUZZY-BASED APPROACHES IN GMDH NETWORKS}

The application of GMDH for structure optimization of fuzzy polynomial neural networks (FPNN) were developed and investigated in numerous works of Witold Pedrycz and his colleagues since 2002 [9-16]. They implemented a like approach as in fuzzy GMDH. In $[9,10]$, W. Pedrycz et al suggested hybrid neural network called fuzzy polynomial neural networks (FPNNs), a hybrid modeling architecture combining polynomial neural networks (PNNs) and fuzzy neural networks (FNNs).

The development of the FPNNs is based on the technologies of computational intelligence (CI). The structure of the FPNN results from a synergistic usage of FNN and PNN. FNNs contribute to the formation of the premise part of the rule-based structure of the FPNN while the consequence part of the FPNN is designed using PNNs. Each PN of the network realizes a polynomial type of partial description (PD) of the mapping between input and out variables: linear, quadratic or modified quadratic. The structure of the PNN is not fixed in advance but it is generated by GMDH to produce dynamic topology of the network.

The authors continued their investigations in the sphere of FPNN and for optimization of it topology suggested genetic algorithms (GAs) [11, 12]. Opposite to the conventional HFPNN which use the GMDH method for structure synthesis, they suggested to apply GA for structure optimization of both FNN and PNN. As a result, genetically optimized HFPNN (gHFPNN) was constructed. The augmented gHFPNN results in a structurally optimized structure and comes with a higher level of flexibility in comparison to the conventional HFPNN. In the sequel, two general optimization mechanisms are explored. First, the structural optimization is realized via GAs whereas the ensuing detailed parametric optimization is carried out in the setting of a standard least square method-based learning.

In $[13,14,15]$, genetically optimized fuzzy relation-based polynomial neural networks were introduced and investigated using information granulation (IG gFRPNN). With the aid of the information granules based on C-Means clustering, the initial location of membership functions were determined and initial values of 
polynomial function being used in the premised and consequence part of the fuzzy rules respectively. The GA-based design procedure being applied at each layer of the IG_gFRPNN leads to the selection of preferred nodes with specific local characteristics (such as the number of input variables, the order of the polynomial, a collection of the specific subset of input variables, and the number of membership functions) available within the network.

In the sequel, the structural optimization is realized via GAs, whereas the ensuing detailed parametric optimization is carried by the standard least square method-based learning. The development of gFRPNN was continued in [16] where the problem of constructing the FRPNN under conditions of high dimensions was considered. Parallel fuzzy polynomial neural networks (PFPNNs) with the aid of heterogeneous partition of the input space were suggested. In the design of the premise part of the rule, a weighted fuzzy clustering method is used not only to realize a non-uniform partition of the input space but to overcome a possible curse of dimensionality. While in the design of consequent part, fuzzy polynomial neural networks are utilized to construct optimal local models (high order polynomials) that describe the relationship between input variables and the output variable within some local region of the input space. Particle swarm optimization (PSO) was employed to adjust the design parameters of parallel fuzzy polynomial neural networks.

The development and investigations of hybrid GMDH- fuzzy neural networks were performed by joint group of scientists in NTUU "KPI" and KTURE (Kharkiv University of Radio-Electronics). In [17, 18], GMDH-wavelet neurofuzzy system was suggested and investigated using advantages of neuro-fuzzy networks and GMDH. In [19], GMDH-cascade neo-fuzzy networks were suggested and investigated in the problem of forecasting. In this work the structure of cascade neo-fuzzy network was constructed using GMDH which enabled to find the structure of network and weights of neurons. In [20], GMDH-neural network with spiking neurons was suggested.

This approach of developing hybrid GMDH-FNN systems was continued in [21] where the authors suggested evolving a hybrid GMDH-neuro fuzzy system. The hybrid system is grounded on both GMDH and the concept of evolving systems that makes it possible to define both optimal parameter values and the best structure in every specific case. The important property of the suggested system is that it does not require any high data volumes to get trained. Adjusting parameters in a parallel fashion gives an option of increasing a processing speed of data handling.

\section{FUZZY GMDH: PRINCIPAL IDEAS AND MATHEMATICAL MODEL CONSTRUCTION}

As it is well-known, the drawbacks of classical GMDH are the followings [3, 4]:

- GMDH utilizes least squared method (LSM) for finding the model coefficients but matrix of linear equations may be close to degenerate and the corresponding solution may appear non-stable and very volatile. Therefore, the special methods for regularization should be used;

- after application of GMDH point-wise estimations are obtained but in many cases it is needed find interval value for coefficient estimates; 
- GMDH does not work in case of incomplete or fuzzy input data.

Therefore, in the last 10 years the new variant of GMDH - fuzzy GMDH was developed and refined which may work with fuzzy input data and is free of classical GMDH drawbacks [3]. As it is well known, GMDH is based on the following principles [1-3]:

1) the principle of multiplicity of models;

2) the principle of external complement which means that the whole sample should be divided into two parts - training subsample and test subsample;

3) the principle of self-organization;

4) the principle of freedom of choice.

Fuzzy GMDH is also based on these principles but construct fuzzy models. Let us consider its main ideas.

In [3-5], the linear interval model regression was considered:

$$
Y=A_{0} Z_{0}+A_{1} Z_{1}+\ldots+A_{n} Z_{n},
$$

where $A_{i}$ is a fuzzy number of triangular form described by pair of parameters $A_{i}=\left(\alpha_{i}, c_{i}\right)$, where $\alpha_{i}$ is interval center, $c_{i}$ is its width, $c_{i} \geq 0$.

Then $Y$ is a fuzzy number with parameters determined as follows:

- the interval center:

$$
\alpha_{y}=\sum \alpha_{i} z_{i}=\alpha^{T} z
$$

- the interval width:

$$
c_{y}=\sum c_{i}\left|z_{i}\right|=c^{T}|z| .
$$

For example, for the partial description of the kind

$$
f\left(x_{i}, x_{j}\right)=A_{0}+A_{1} x_{i}+A_{2} x_{j}+A_{3} x_{i} x_{j}+A_{4} x_{i}^{2}+A_{5} x_{j}^{2}
$$

it is necessary to assign in the general model (1),

$$
z_{0}=1, z_{1}=x_{i}, z_{2}=x_{j}, z_{3}=x_{i} x_{j}, z_{4}=x_{i}^{2}, z_{5}=x_{j}^{2} .
$$

Let the training sample be $\left\{z_{1}, z_{2}, \ldots, z_{M}\right\},\left\{y_{1}, y_{2}, \ldots, y_{M}\right\}$. Then for the model (1) to be adequate it is necessary to find such parameters $\left(\alpha_{i}, c_{i}\right) i=\overline{1, n}$ satisfying the following inequalities:

$$
\left\{\begin{array}{l}
\alpha^{T} z_{k}-c^{T}\left|z_{k}\right| \leq y_{k}, \quad k=\overline{1, M} . \\
\alpha^{T} z_{k}+c^{T}\left|z_{k}\right| \geq y_{k},
\end{array}\right.
$$

Let us formulate the basic requirements for the linear interval model of partial description of a kind (1).

It is necessary to find such values of the parameters $\left(\alpha_{i}, c_{i}\right)$ of fuzzy coefficients for which:

1) real values of observed outputs $y_{k}$ would drop in the estimated interval for $Y_{k}$;

2) the total width of the estimated interval for all sample points would be minimal. 
These requirements lead to the following linear programming problem [3, 4]:

$$
\min \left(C_{0} M+C_{1} \sum_{k=1}^{M}\left|x_{k i}\right|+C_{2} \sum_{k=1}^{M}\left|x_{k j}\right|+C_{3} \sum_{k=1}^{M}\left|x_{k i} x_{k j}\right|+C_{4} \sum_{k=1}^{M}\left|x_{k i}^{2}\right|+C_{5} \sum_{k=1}^{M}\left|x_{k j}^{2}\right|\right)
$$

under constraints:

$$
\begin{gathered}
a_{0}+a_{1} x_{k i}+a_{2} x_{k j}+a_{3} x_{k i} x_{k j}+a_{4} x_{k i}^{2}+a_{5} x_{k j}^{2}-\left(C_{0}+C_{1}\left|x_{k i}\right|+C_{2}\left|x_{k j}\right|+\right. \\
\left.+C_{3}\left|x_{k i} x_{k j}\right|+C_{4}\left|x_{k i}^{2}\right|+C_{5} \mid x_{k j}^{2}\right) \leq y_{k}, \\
a_{0}+a_{1} x_{k i}+a_{2} x_{k j}+a_{3} x_{k i} x_{k j}+a_{4} x_{k i}^{2}+a_{5} x_{k j}^{2}+\left(C_{0}+C_{1}\left|x_{k i}\right|+C_{2}\left|x_{k j}\right|+\right. \\
\left.+C_{3}\left|x_{k i} x_{k j}\right|+C_{4}\left|x_{k i}^{2}\right|+C_{5}\left|x_{k j}^{2}\right|\right) \geq y_{k},
\end{gathered}
$$

where $C_{p} \geq 0, \quad p=0,5, k=\overline{1, M}$ and $k$ is an index of a point.

As we can easily see the task (2) - (4) is linear programming (LP) problem. However, the inconvenience of the model (2) - (4) for the application of standard LP methods is that there are no constraints of non-negativity for variables $a_{i}$. Therefore for its solution it is reasonable to pass to the dual LP problem by introducing dual variables $\left\{\delta_{k}\right\}$ and $\left\{\delta_{k+M}\right\}, k=\overline{1, M}$. Using simplex-method for the dual problem and after finding the optimal values for the dual variables $\left\{\delta_{k}\right\}$, the optimal solutions $\left(a_{i}, c_{i}\right)$ of the initial direct problem will be also found.

\section{THE DESCRIPTION OF FUZZY GMDH ALGORITHM}

Let us present the brief description of the algorithm FGMDH [3, 4].

1. Choose the general model type by which the sought dependence will be described.

2. Choose the external criterion of optimality (criterion of regularity or unbiasedness).

3. Choose the type of partial descriptions (for example, linear or quadratic one).

4. Divide the sample into training $N_{\text {train }}$ and test $N_{\text {test }}$ subsamples.

5. Put zero values to the counter of model number $\mathrm{k}$ and to the counter of layers $r$ (iterations number).

6. Generate a new partial model $f_{k}(1)$ using the training sample. Solve the LP problem (2) - (4) and find the values of parameters $\alpha_{i}, c_{i}$.

7. Calculate using test sample the value of external criterion $\left(N_{u b k}^{(r)}\right.$ or $\left.\delta_{k}^{(2)}(r)\right)$.

8. $k=k+1$. If $k>C_{N}^{2}$ for $r=1$ or $k>C_{F}^{2}$ for $r>1$, then $k=1, r=r+1$ and go to step 9 , otherwise go to step 6 .

9. Calculate the best value of the criterion for models of r-th iteration. If $r=1$, then go to step 6 otherwise, go to step 10 . 
10. If $\left|N_{u b}(r)-N_{u b}(r-1)\right| \leq \varepsilon$ or $\delta_{k}^{(2)}(r) \geq \delta_{k-1}^{(2)}(r)$, then go to 11 , otherwise select $F$ best models and assigning $r=r+1, k=1$, go to step 6 and execute $(r+1)$-th iteration.

11. Select the best model out of models of the previous layer (iteration) using external criterion.

\section{ANALYSIS OF DIFFERENT MEMBERSHIP FUNCTIONS}

In the first paper devoted to fuzzy GMDH [3], the triangular membership functions (MF) were considered. But as fuzzy numbers may also have the other kinds of MF it is important to consider the other classes of MF in the problems of modeling using FGMDH. In [4], fuzzy models with Gaussian and bell-shaped MF were investigated.

Consider a fuzzy set with MF of the form:

$$
\mu_{B}(x)=e^{-\frac{1}{2} \frac{(x-1)^{2}}{c^{2}}} .
$$

Let the linear interval model for partial description of FGMDH take the form (1). Then the problem is to find such fuzzy numbers $B_{i}$ with parameters $\left(a_{i}, c_{i}\right)$ that:

- the observation $y_{k}$ would belong to a given estimate interval for the set $Y(k)$ with degree not less than $\alpha, 0<\alpha<1$;

- the width of the estimated interval of the degree $\alpha$ would be minimal;

In $[4,6]$ it was shown that the problem of finding optimal fuzzy model will be finally transformed to the following LP problem:

$$
\min \left(C_{0} M+C_{1} \sum_{k=1}^{M}\left|x_{k i}\right|+C_{2} \sum_{k=1}^{M}\left|x_{k j}\right|+C_{3} \sum_{k=1}^{M}\left|x_{k i} x_{k j}\right|+C_{4} \sum_{k=1}^{M}\left|x_{k i}^{2}\right|+C_{5} \sum_{k=1}^{M}\left|x_{k j}^{2}\right|\right) ;
$$

under constraints:

$$
\left.\begin{array}{l}
a_{0}+a_{1} x_{k i}+\ldots+a_{5} x_{k j}^{2}+\left(C_{0}+C_{1}\left|x_{k i}\right|+\ldots+C_{5}\left|x_{k j}^{2}\right|\right) \sqrt{-2 \ln \alpha} \geq y_{k}, \\
a_{0}+a_{1} x_{k i}+\ldots+a_{5} x_{k j}^{2}-\left(C_{0}+C_{1}\left|x_{k i}\right|+\ldots+C_{5}\left|x_{k j}^{2}\right|\right) \sqrt{-2 \ln \alpha} \leq y_{k},
\end{array}\right\} k=\overline{1, M} .
$$

To solve this problem like in the case of triangular MF it is reasonable to pass to the dual LP problem of the form:

$$
\max \left(\sum_{k=1}^{M} y_{k} \delta_{k+\mathrm{M}}-\sum_{k=1}^{M} y_{k} \delta_{k}\right)
$$

with constraints of equalities and inequalities:

$$
\begin{gathered}
\sum_{k=1}^{M} \delta_{k+\mathrm{M}}-\sum_{k=1}^{M} \delta_{k}=0 ; \\
\sum_{k=1}^{M} X_{k i} \delta_{k+M}-\sum_{k=1}^{M} X_{k i_{i}} \delta_{k}=0
\end{gathered}
$$




$$
\begin{aligned}
& \sum_{k=1}^{M} X_{k j}{ }^{2} \delta_{k+M}-\sum_{k=1}^{M} X_{k_{j}}^{2} \delta_{k}=0 \\
& \sum_{k=1}^{M} \delta_{k}+\sum_{k=1}^{M} \delta_{k+M} \leq \frac{M}{\sqrt{-2 \ln \alpha}}, \\
& \sum_{k=1}^{M}\left|X_{k i}\right| \delta_{k+M}+\sum_{k=1}^{M}\left|X_{k i}\right| \delta_{k} \leq \frac{\sum_{k=1}^{M}\left|X_{k i}\right|}{\sqrt{-2 \ln \alpha}}, \\
& \sum_{k=1}^{M}\left|X_{k j}^{2}\right| \delta_{k+M}+\sum_{k=1}^{M}\left|X_{k j}^{2}\right| \delta_{k} \leq \frac{\sum_{k=1}^{M}\left|X_{k j}^{2}\right|}{\sqrt{-2 \ln \alpha}}, \\
& \delta_{k} \geq 0, k=\overline{1,2 M} \text {. }
\end{aligned}
$$

Analyzing the dual LP program (5)-(10), it is easy to notice that this problem is always solvable as there is trivial solution $\delta_{k}=1, k=\overline{1,2 M}$. Therefore the initial problem is also always solvable with any data.

Thus, fuzzy GMDH allows constructing fuzzy models and has the following advantages:

1. The problem of optimal model determination is transferred to the problem of linear programming which is always solvable.

2. There is so called interval regression model built as the result of the method performance.

\section{FUZZY GMDH WITH DIFFERENT PARTIAL DESCRIPTIONS: ORTHOGONAL POLYNOMIALS}

As it is well known from the general GMDH theory, models-pretenders are generated in this method on the base of so called partial descriptions being elementary models of two variables. Usually as partial descriptions linear or quadratic polynomials are used. The alternative to this class of those elementary models is application of orthogonal polynomials. The choice of such polynomials as partial descriptions is determined by the following advantages:

- Owing to orthogonal property, the calculation of polynomial coefficients which approximate the simulated process goes faster than for non-orthogonal polynomials.

- The coefficients of polynomial approximating equation do not depend on the degree of initial polynomial model so if a priori the real polynomial degree is not known we may check the polynomials of various degrees and by this property the coefficients obtained for polynomials of lower degrees remain the same after transfer to higher polynomial degrees. This property is the most important during investigation of real degree of approximating polynomial when solving applied problems.

One of the properties of orthogonal polynomials widely used in this work is the property of almost equal errors. Owing to this the very large errors do not 
happen, on the contrary, in most cases the error values are small. Therefore the damping of approximation errors occurs. The application of orthogonal polynomials as partial descriptions in FGMDH was suggested and investigated in $[5,6]$.

\section{Chebyshev's orthogonal polynomials}

Chebyshev's orthogonal polynomials in general case have the following form [5]:

$$
F_{v}(\xi)=T_{v}(\xi)=\cos (v \arccos \xi),-1 \leq \xi \leq 1 .
$$

These polynomials have the following orthogonality property:

$$
\int_{-1}^{1} \frac{T_{\mu}(\xi) T_{v}(\xi) d \xi}{\sqrt{1-\xi^{2}}}=\left\{\begin{array}{l}
0 \text { if } \mu \neq \xi ; \\
\frac{\pi}{2} \text { if } \mu=\xi \neq 0 ; \\
\pi \text { if } \mu=\xi=0,
\end{array}\right.
$$

where $\sqrt{1-\xi^{2}}$ is a weighting coefficient $\omega(\xi)$ in the equation (11).

The approximating Chebyshev's orthogonal polynomial for $\bar{y}$ is obtained on the base of function $S$ minimization:

$$
S=\int_{-1}^{1} \omega(\xi)\left(y(\xi)-\sum_{i=0}^{m} b_{i} T_{i}(\xi)\right)^{2} d \xi .
$$

From (12) it follows that

$$
b_{k}=\left\{\begin{array}{cl}
\frac{1}{\pi} \int_{-1}^{1} \frac{y(\xi)}{\sqrt{1-\xi^{2}}} d \xi, & k=0 ; \\
\frac{2}{\pi} \int_{-1}^{1} \frac{y(\xi) T_{k}(\xi)}{\sqrt{1-\xi^{2}}} d \xi, & k \neq 0 .
\end{array}\right.
$$

Hence, the approximating equation is obtained in the form:

$$
\bar{y}(\xi)=\sum_{k=0}^{m} b_{k} T_{k}(\xi) .
$$

As it may be readily seen from the presented expressions, coefficient $b_{k}$ (13) does not depend on choice of degree $m$. Thus, the variable $m$ does not demand recalculation of $b_{j} \forall j \leq m$ while such recalculation is necessary for nonorthogonal approximation.

The best approximating degree $m^{*}$ may be obtained on the base of hypothesis that investigation results $y(i), i=1,2, \ldots, r$, have independent Gaussian distribution in the bounds of some polynomial function $\bar{y}$ of certain degree, e.g. $m^{*}+\mu$, where

$$
\bar{y}_{m^{*}+\mu}\left(x_{i}\right)=\sum_{j=0}^{m^{*}+\mu} b_{j} x_{i}^{j},
$$

and the dispersion $\sigma^{2}$ of distribution $(y-\bar{y})$ does not depend on $\mu$. 
It is clear that for a very small $m(m=0,1,2, \ldots) \sigma_{m}^{2}$ decreases as $m$ grows.

As in accordance with the previously formulated hypothesis dispersion does not depend on $m$, the best degree $m^{*}$ is the minimal $m$ for which $\sigma_{m} \cong \sigma_{m+1}$.

For determining $m^{*}$ it is necessary to calculate the approximating polynomials of various degrees. As coefficients $b_{j}$ in the equation do not depend on $m$, the determination of the best degree of polynomial is accelerated.

Let we have the forecasted variable $Y$ and input variables $x_{1}, x_{2}, \ldots x_{n}$. Let us search the relation between them in the following form

$$
Y=A_{1} f_{1}\left(x_{1}\right)+A_{2} f_{2}\left(x_{2}\right)+\ldots+A_{n} f_{n}\left(x_{n}\right),
$$

where $A_{i}$ is a fuzzy number of triangular type given as $A_{i}=\left(\alpha_{i}, c_{i}\right)$, functions $f_{i}$ are determined as follows $[5,6]$ :

$$
f_{i}\left(x_{i}\right)=\sum_{j=0}^{m_{i}} b_{i j} T_{j}\left(x_{i}\right) .
$$

The degree $m_{i}$ of function $f_{i}$ is determined using hypothesis defined in the preceding section. Denoting $z_{i}=f_{i}\left(x_{i}\right)$, we get a linear interval model in classical form.

\section{Investigation of Trigonometric Polynomials as Partial Descriptions}

Let a function $f(x)$ be periodic with period $2 \pi$ defined at the interval $[-\pi, \pi]$, and its derivative $f^{\prime}(x)$ is also defined at $[-\pi, \pi]$. Then the following equality holds

where

$$
S(x)=f(x) \forall x \in[-\pi, \pi],
$$

$$
S(x)=\frac{a_{0}}{2}+\sum_{j=1}\left(a_{j} \cos (j x)+b_{j} \sin (j x)\right) .
$$

Coefficients $a_{j}, b_{j}$ are calculated by Euler formulas:

$$
a_{j}=\frac{1}{\pi} \int_{-\pi}^{\pi} f(x) \cos (j x) d x ; \quad b_{j}=\frac{1}{\pi} \int_{-\pi}^{\pi} f(x) \sin (j x) d x .
$$

Definition. A trigonometric polynomial of the degree $M$ is called the expression:

$$
T_{M}(x)=\frac{a_{0}}{2}+\sum_{j=1}^{M}\left(a_{j} \cos (j x)+b_{j} \sin (j x)\right) .
$$

The following theorem is true stating that exists such $M, 2 M<N$, which minimizes the expression [6]:

$$
\sum_{j=1}^{N}\left(f\left(x_{i}\right)-T_{M}\left(x_{i}\right)\right)^{2}
$$

Hence the coefficients of corresponding trigonometric polynomial are determined by formulas: 


$$
a_{j}=\frac{2}{N} \sum_{i=1}^{N} f\left(x_{i}\right) \cos \left(j x_{i}\right) ; b_{j}=\frac{2}{N} \sum_{i=1}^{N} f\left(x_{i}\right) \sin \left(j x_{i}\right) .
$$

Let it be the variable $Y$ to be forecasted and input variables $x_{1}, x_{2}, \ldots, x_{n}$. Let us search the dependence among them in the form:

$$
Y=A_{1} f_{1}\left(x_{1}\right)+A_{2} f_{2}\left(x_{2}\right)+\ldots+A_{n} f_{n}\left(x_{n}\right),
$$

where $A_{i}$ is a fuzzy number of triangular type given as $A_{i}=\left(\alpha_{i}, c_{i}\right)$, functions $f_{i}$ are determined in such a way:

$$
f_{i}\left(x_{i}\right)=T_{M_{i}}\left(x_{i}\right) .
$$

The degree $M_{i}$ of a function $f_{i}$ is determined by the theorem described in the preceding section. Therefore if to assign $z_{i}=f_{i}\left(x_{i}\right)$, the linear interval model will be obtained in its classical form.

\section{ADAPTATION OF FUZZY GMDH MODELS}

While forecasting by self-organizing methods (fuzzy GMDH, in particular), the problem arises regarding the necessity of huge amount of repetitive calculations in case of the training sample size increase or while forecasting in real time when it is needed to correct the obtained model in accordance with new available data. Taking into account new information obtained while forecasting, adaptation may be done by two approaches. The first one is to correct parameters of a forecasting model with new data assuming that model structure did not change. The second approach consists in adaptation of not only model parameters but its optimal structure as well.

This way demands the repetitive use of full GMDH algorithm and is connected with huge volume of calculations. The second approach is used if adaptation of parameters does not provide good forecast and the new real output values do not drop in the calculated interval for its estimate.

In our consideration, the first approach is used based on adaptation of FGMDH model parameters to new available data. Here the recursive identification methods are preferably used, especially the recursive least squared method (LSM). In this method the parameters estimations on the next step are determined on the base of estimates on the previous step, model error and some information matrix which is modified during all estimation process and therefore contains data which may be used at the next steps of adaptation process [5].

Hence, model coefficients adaptation will be simplified substantially. If to store information matrix obtained while identification of optimal model using fuzzy GMDH, then for model parameters adaptation it will be enough to fulfill only one iteration by recursive LSM method.

\section{The Application of Recurrent LSM for Model Coefficients Adaptation}

Consider the following model:

$$
y(k)=\theta^{T} \Psi(k)+v(k),
$$

where $y(k)$ is a dependent (output) variable, $\Psi(k)$ is a measurements vector, $v(k)$ are random disturbances, $\theta$ is a parameters vector to be estimated. 
The parameters estimate $\theta$ at the step $N$ is performed due to such formula $[5,6]$ :

$$
\widehat{\theta}(N)=\hat{\theta}(N-1)+\gamma(N)\left[y(N)-\widehat{\theta}^{T}(N-1) \Psi(N)\right],
$$

where $\gamma(N)$ is a coefficients vector which is determined by formula:

$$
\gamma(N)=\frac{P(N-1) \Psi(N)}{1+\Psi^{T}(N) P(N-1) \Psi(N)},
$$

where $P(N-1)$ is so-called "information matrix" determined by formula:

$$
P(N-1)=P(N-2)-\frac{P(N-2) \Psi(N-1) \Psi^{T}(N-1) P(N-2)}{1+\Psi^{T}(N-1) P(N-2) \Psi(N-1)} .
$$

As one can see from (14), the information matrix may be obtained independently on parameters estimation process and parallel to it. The adaptation of two parameter vectors $\theta_{1}^{T}=\left[\alpha_{1}, \ldots, \alpha_{m}\right], \theta_{2}^{T}=\left[C_{1}, \ldots, C_{m}\right]$, is performed using the formulas [35]:

$$
\begin{gathered}
\hat{\theta}_{1}(N)=\widehat{\theta}_{1}(N-1)+\gamma_{1}(N)\left[y(N)-\widehat{\theta}_{1}^{T}(N-1) \Psi_{1}(N)\right] ; \\
\widehat{\theta}_{2}(N)=\widehat{\theta}_{2}(N-1)+\gamma_{2}(N)\left[y_{c}(N)-\widehat{\theta}_{2}^{T}(N-1) \Psi_{2}(N)\right] ; \\
y_{c}(N)=\left|y(N)-\theta_{1}^{T}(N-1) \Psi_{1}(N)\right|,
\end{gathered}
$$

where $\Psi_{1}^{T}=\left[z_{1}, \ldots, z_{m}\right] ; \quad \Psi_{2}^{T}=\left[\left|z_{1}\right|, \ldots,\left|z_{m}\right|\right]$.

\section{APPLICATION OF GMDH FOR FORECASTING STOCK EXCHANGE PROCESSES}

Consider the application of GMDH and fuzzy GMDH for forecasting at the stock exchange NYSE. As input variables, the following stock prices at NYSE were chosen: close prices of companies Hess Corporation, Repsol YPF, S.A. (ADR), Eni S.p.A. (ADR), Exxon Mobil Corporation, Chevron Corporation, and Total S.A. (ADR) [7]. As an output variable, close stock prices of British Petroleum BP plc (ADR) were chosen.

The second problem was forecasting industrial index Dow-Jones Average. As the input variables in this problem there were taken close stock prices of the following companies which form it: American Express Company, Bank of America, Coca-cola, McDonald's, Microsoft Corp., Johnson\&Johnson, Intel Corp.

The training sample data were taken in the period since 20 September to 14 November 2011. For the test sample, data of Dow Jones Industrial Average were taken since 15 November 2011 year to 17 November 2011 year [7].

For the experimental investigations, classical GMDH and fuzzy GMDH were used. For these experiments, the percentage of training sample variants was chosen $50 \%, 70 \%, 90 \%$. Freedom of choice $F$ was taken 5 and 6 .

For fuzzy GMDH, triangular, Gaussian and bell-shaped membership functions were used. For Gaussian and bell-shaped membership functions, the following level values were taken: $a=0,3 ; 0,5 ; 0,7 ; 0,9$. 
To construct models, the following four partial description types were used:

- a linear model of the form:

$$
f\left(x_{i}, x_{j}\right)=A_{0}+A_{1} x_{i}+A_{2} x_{j}
$$

- a squared model:

$$
f\left(x_{i}, x_{j}\right)=A_{0}+A_{1} x_{i}+A_{2} x_{j}+A_{3} x_{i} x_{j}+A_{4} x_{i}^{2}+A_{5} x_{j}^{2} ;
$$

- Fourier polynomial of the first degree:

$$
T_{1}=\frac{a_{0}}{2}+a_{1} \sin (x)+b_{1} \cos (x)
$$

- Chebyshev's polynomial of the second degree:

$$
f\left(x_{i}, x_{j}\right)=A_{0}+A_{1} x_{i}+A_{2}\left(x_{j}^{2}-1\right),
$$

where $A_{j}$ is a fuzzy number with triangular, Gaussian or bell-shaped membership function.

For forecasts accuracy analysis, the following criteria of the forecast quality were used: MSE for test sample, MSE for full sample, MAPE for full and test sample, Durbin-Watson criterion (DW), R-square, Akaike criterion (AIC), Bayes information criterion (BIC), and Shwartz criterion (SC). These criteria values were calculated for each forecast step using the test sample. The results of forecast for BP plc (ADR) shares are presented in the table 1. and for Dow Jones Industrial Average in the table 2.

Table 1. Forecast quality criteria at each forecast step by fuzzy GMDH for shares BP plc (ADR) closing prices

\begin{tabular}{|c|c|c|c|c|}
\hline $\begin{array}{c}\text { Step of fore- } \\
\text { cast }\end{array}$ & \multirow{2}{*}{ Criterion } & \multicolumn{3}{|c|}{ Percentage of training sample } \\
\cline { 2 - 5 } & MSE test & $50 \%$ & $70 \%$ & $90 \%$ \\
\hline \multirow{4}{*}{1} & MSE & 0,748864 & 0,612827 & 0,485599 \\
\cline { 2 - 5 } & MAPE test & 2,041366 & 2,065155 & 1,386096 \\
\cline { 2 - 5 } & MAPE & 1,452656 & 1,723657 & 1,505965 \\
\cline { 2 - 5 } & DW & 0,686478 & 1,763043 & 1,839065 \\
\cline { 2 - 5 } & R-square & 1,064526 & 0,938058 & 1,008042 \\
\cline { 2 - 5 } & AIC & 2,456985 & 2,228267 & 2,053752 \\
\cline { 2 - 5 } & BIC & $-3,268440$ & $-3,063$ & $-2,892253$ \\
\hline \multirow{4}{*}{2} & SC & 2,506693 & 2,277 & 2,103459 \\
\cline { 2 - 5 } & MSE test & 1,256828 & 1,871883 & 2,440575 \\
\cline { 2 - 5 } & MSE & 0,728499 & 0,989650 & 0,795699 \\
\cline { 2 - 5 } & MAPE test & 2,072085 & 2,367006 & 2,426847 \\
\cline { 2 - 5 } & MAPE & 1,468987 & 1,845798 & 1,656084 \\
\cline { 2 - 5 } & DW & 0,686478 & 1,795021 & 1,839065 \\
\cline { 2 - 5 } & R-square & 1,064526 & 0,874163 & 1,008042 \\
\cline { 2 - 5 } & AIC & 2,456985 & 2,147717 & 2,053752 \\
\hline & BIC & $-3,268440$ & $-2,986448$ & $-2,892253$ \\
\cline { 2 - 5 } & SC & 2,506693 & 2,197424 & 2,103459 \\
\hline
\end{tabular}


The flow charts of forecasts for shares BP plc (ADR) obtained by fuzzy GMDH are presented on Fig. 1 for 2 steps ahead with $F=6$, the training sample size $70 \%$ and Gaussian MF, significance level $a=0,7$.

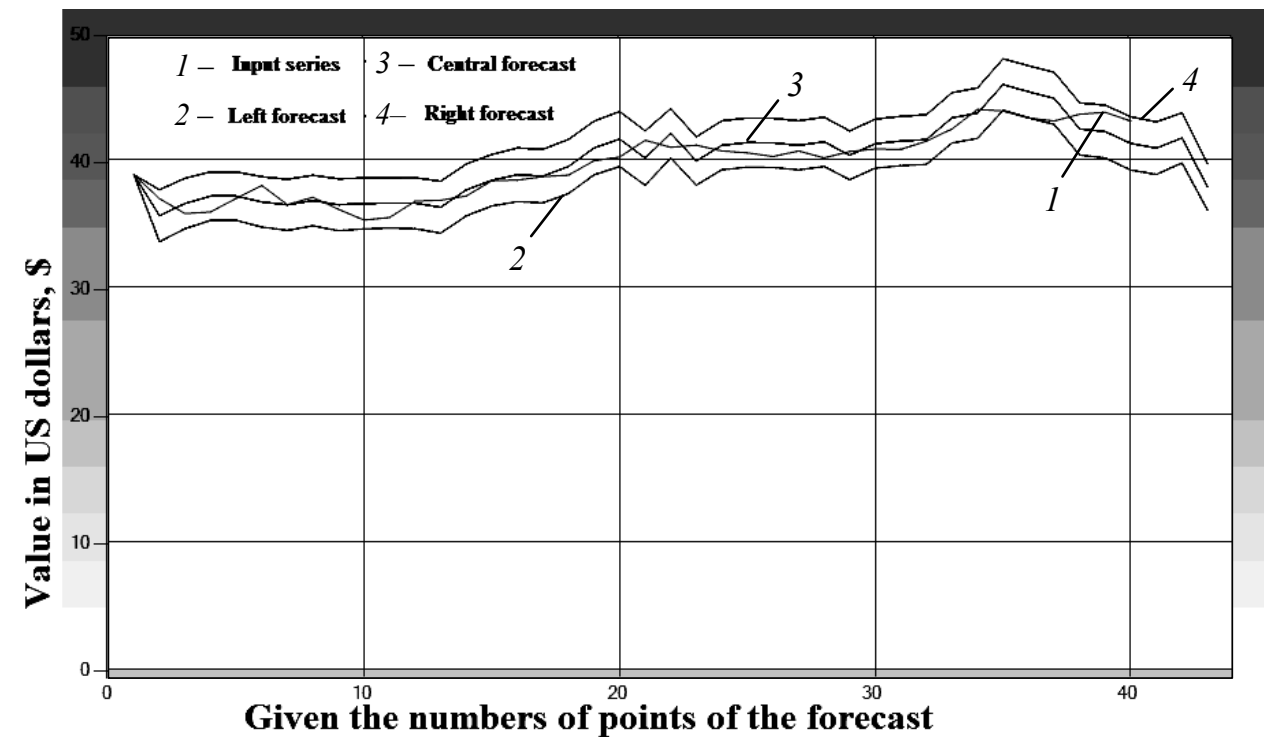

Fig. 1. Forecast results for BP plc (ADR) shares by FGMDH with quadratic partial descriptions, 3 steps ahead

Table 2. Forecast quality criteria by fuzzy GMDH for Dow Jones Industrial Average

\begin{tabular}{|c|c|c|c|c|}
\hline \multirow{2}{*}{ Step of forecast } & \multirow{2}{*}{ Criterion } & \multicolumn{3}{|c|}{ Percentage of training sample } \\
\cline { 2 - 5 } & & $50 \%$ & $70 \%$ & $90 \%$ \\
\hline \multirow{4}{*}{1} & MSE test & 40494,427 & 33109,754 & 40286,725 \\
\cline { 2 - 5 } & MSE & 26900,763 & 62553,804 & 26432,081 \\
\cline { 2 - 5 } & MAPE test & 1,462066 & 1,363148 & 1,405372 \\
\cline { 2 - 5 } & MAPE & 1,149183 & 1,809130 & 1,191039 \\
\cline { 2 - 5 } & DW & 1,917430 & 1,013536 & 1,862203 \\
\cline { 2 - 5 } & R-square & 0,990922 & 0,804774 & 0,849329 \\
\cline { 2 - 5 } & AIC & 12,753417 & 13,551054 & 12,729001 \\
\cline { 2 - 5 } & BIC & $-7,360808$ & $-7,482613$ & $-7,356959$ \\
\cline { 2 - 5 } & SC & 12,796073 & 13,593710 & 12,771656 \\
\hline \multirow{4}{*}{2} & MSE test & 41546,293 & 31602,995 & 43907,693 \\
\cline { 2 - 5 } & MSE & 27793,341 & 61328,007 & 32746,387 \\
\cline { 2 - 5 } & MAPE test & 1,481603 & 1,374860 & 1,521950 \\
\cline { 2 - 5 } & MAPE & 1,167355 & 1,819287 & 1,280730 \\
\cline { 2 - 5 } & DW & 1,917020 & 1,013536 & 1,669879 \\
\cline { 2 - 5 } & R-square & 0,989976 & 0,804774 & 0,811488 \\
\cline { 2 - 5 } & AIC & 12,753849 & 13,551054 & 12,935378 \\
\cline { 2 - 5 } & BIC & $-7,360875$ & $-7,482613$ & $-7,389255$ \\
\cline { 2 - 5 } & SC & 12,796504 & 13,593710 & 12,978034 \\
\hline
\end{tabular}


Forecasts by fuzzy GMDH for Dow Jones I.A. shares are presented on Fig. 2, 3 steps ahead, $F=6$, the training sample size $70 \%$ and Gaussian MF, significance level $a=0,7$.

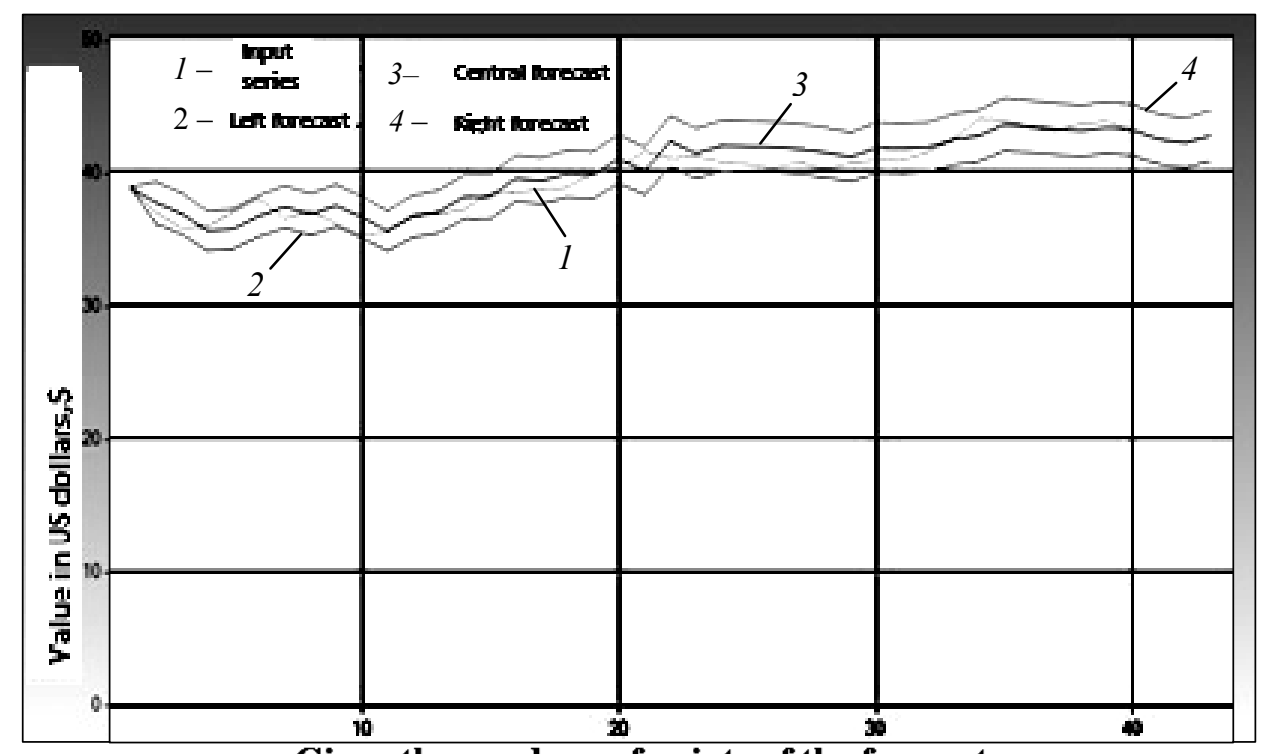

Given the numbers of points of the forecast

Fig. 2. Index Dow Jones I.A. forecast results at 3 steps ahead with FGMDH

Further experiments for forecasting share prices of BP plc (ADR) and Dow Jones I.A. were carried out with application of GMDH and fuzzy GMDH with different partial descriptions: linear model, squared model, Chebyshev's polynomials and Fourier polynomials and with application of cascade neo-fuzzy neural networks as well [7].

The final experimental results of forecasts at 1,2 and 3 steps ahead with aforesaid methods for share prices of British Petroleum BP plc (ADR) are presented in the table 3 and for index Dow Jones Industrial Average in the table 4.

Table 3. Comparative forecasting results for BP plc (ADR) share prices

\begin{tabular}{|c|c|c|c|c|c|c|c|c|c|}
\hline \multirow{2}{*}{$\begin{array}{c}\text { Step } \\
\text { of } \\
\text { fore- } \\
\text { cast }\end{array}$} & \multirow{2}{*}{ Criteria } & \multicolumn{3}{|c|}{$\begin{array}{r}\text { Forecast results for GMDH } \\
\text { Partial description (PD) }\end{array}$} & \multicolumn{3}{|c|}{$\begin{array}{c}\text { Forecast results for fuzzy GMDH } \\
\text { Partial description }\end{array}$} \\
\cline { 3 - 10 } & Linear & $\begin{array}{c}\text { Quad- } \\
\text { ratic }\end{array}$ & $\begin{array}{c}\text { Fourier } \\
\text { poly- } \\
\text { nomial }\end{array}$ & $\begin{array}{c}\text { Chebyshev's } \\
\text { polynomial }\end{array}$ & Linear & $\begin{array}{c}\text { Quad- } \\
\text { ratic }\end{array}$ & $\begin{array}{c}\text { Fourier } \\
\text { poly } \\
\text { nomial }\end{array}$ & $\begin{array}{c}\text { Chebyshev's } \\
\text { polynomial }\end{array}$ \\
\hline \multirow{2}{*}{1} & MSE & 0,285 & 1,905 & 0,859 & 0,365 & 0,481 & 0,130 & 1,691 & 0,757 \\
\cline { 2 - 10 } & MAPE & 1,034 & 1,965 & 1,624 & 1,114 & 1,374 & 0,813 & 2,960 & 1,459 \\
\hline \multirow{2}{*}{2} & MSE & 0,425 & 3,090 & 1,094 & 0,366 & 0,498 & 0,150 & 1,742 & 1,029 \\
\cline { 2 - 10 } & MAPE & 1,227 & 2,916 & 1,814 & 1,115 & 1,481 & 0,818 & 2,977 & 1,584 \\
\hline \multirow{2}{*}{3} & MSE & 0,675 & 4,978 & 2,144 & 0,523 & 0,572 & 0,308 & 2,183 & 1,505 \\
\cline { 2 - 10 } & MAPE & 1,496 & 4,434 & 2,050 & 1,320 & 1,494 & 0,908 & 3,024 & 1,681 \\
\hline
\end{tabular}

The best results were obtained by fuzzy GMDH with quadratic PD, 70\% training sample and Gaussian MF. The worst results gives Fourier polynomials as PD. The both GMDH methods, classical and fuzzy, have shown the high forecast 
accuracy. If to compare the accuracy of both methods with linear partial descriptions, then linear model by GMDH has shown more accurate results. But with all used PD most accurate forecasts were obtained using fuzzy GMDH with quadratic partial descriptions.

Table 4. Comparative forecasting results for index Dow Jones I.A.

\begin{tabular}{|c|c|c|c|c|c|c|c|c|c|}
\hline \multirow{2}{*}{$\begin{array}{c}\text { Step } \\
\text { of } \\
\text { fore- } \\
\text { cast }\end{array}$} & \multirow{2}{*}{ Criteria } & \multicolumn{3}{|c|}{$\begin{array}{c}\text { Forecast results for GMDH } \\
\text { Partial descriptions }\end{array}$} & \multicolumn{3}{|c|}{$\begin{array}{c}\text { Forecast results for fuzzy GMDH } \\
\text { Partial descriptions }\end{array}$} \\
\cline { 3 - 11 } & Linear & $\begin{array}{c}\text { Quad- } \\
\text { ratic }\end{array}$ & $\begin{array}{c}\text { Fourier } \\
\text { poly- } \\
\text { nomial }\end{array}$ & $\begin{array}{c}\text { Chebyshev's } \\
\text { polynomial }\end{array}$ & Linear & $\begin{array}{c}\text { Quad- } \\
\text { ratic }\end{array}$ & $\begin{array}{c}\text { Fourier } \\
\text { polyno- } \\
\text { mial }\end{array}$ & $\begin{array}{c}\text { Chebyshev's } \\
\text { polynomial }\end{array}$ \\
\hline \multirow{2}{*}{1} & MSE & 26900 & 38225 & 40142 & 23818 & 25176 & 21332 & 42205 & 24464 \\
\cline { 2 - 11 } & MAPE & 1,149 & 1,298 & 1,445 & 1,111 & 1,137 & 1,046 & 1,487 & 1,125 \\
\hline \multirow{2}{*}{2} & MSE & 27793 & 39460 & 40930 & 23978 & 25793 & 223491 & 59059 & 24767 \\
\cline { 2 - 11 } & MAPE & 1,167 & 1,322 & 1,445 & 1,119 & 1,143 & 1,098 & 1,614 & 1,144 \\
\hline \multirow{2}{*}{3} & MSE & 37306 & 50471 & 41720 & 27337 & 29782 & 38291 & 63900 & 24910 \\
\cline { 2 - 11 } & MAPE & 1,230 & 1,386 & 1,460 & 1,157 & 1,176 & 1,099 & 1,623 & 1,160 \\
\hline
\end{tabular}

The best results were obtained by fuzzy GMDH with quadratic partial descriptions with bell-shaped membership functions and 50\% training sample size. The worst results were obtained with Fourier polynomial as partial descriptions. The use of Chebyshev's polynomial as PD in classical GMDH has shown the best results. In fuzzy GMDH the most accurate estimates were obtained with linear and quadratic PD.

\section{FGMDH MODEL WITH FUZZY INPUT DATA}

\section{FGMDH Model Construction with Fuzzy Inputs}

Let us consider the generalization of fuzzy GMDH for case when input data are also fuzzy. Then a linear interval regression model takes the following form $[6,8]$ :

$$
Y=A_{0} Z_{0}+A_{1} Z_{1}+\ldots+A_{n} Z_{n},
$$

where $A_{i}$ is a fuzzy number of triangular shape with parameters $A_{i}=\left(\underline{A}_{i}, \breve{A}_{i}, \bar{A}_{i}\right)$, where $\breve{A}_{i}$ is a center of the interval, $\bar{A}_{i}$ - its upper border, and $\underline{A}_{i}$ - lower border.

Consider the case of symmetrical membership function for parameters $A_{i}$, so they can be described by the pair of parameters $\left(\breve{A}_{i}, c_{i}\right), c_{i}$-interval width, $c_{i} \geq$ 0: $A_{i}=\breve{A}_{i}-c_{i}, A_{i}=\breve{A}_{i}+c_{i}$.

Let $Z_{i}$ be also a fuzzy number of triangular shape defined as $\left(\underline{Z}_{i}, \breve{Z}_{i}, \overline{Z_{i}}\right)$, $Z_{i}$ is a lower border, $\breve{Z}_{i}$ is a center, and $\bar{Z}_{i}$ is an upper border of the fuzzy number.

Then $Y$ is a fuzzy number which parameters are defined as follows:

- the center of the interval $\breve{y}=\sum a_{i} \breve{Z}_{i}$;

- the deviation in the left part of the membership function:

$$
\breve{y}-\underline{y}=\sum\left(a_{i}\left(\breve{Z}_{i}-\underline{Z}_{i}\right)+c_{i}\left|\breve{Z}_{i}\right|\right) \text {; }
$$


- the lower border of the interval

$$
\underline{y}=\sum\left(a_{i} \underline{Z}_{i}-c_{i} \mid \breve{Z}_{i}\right) \text {; }
$$

- the deviation in the right part of the membership function:

$$
\bar{y}-\breve{y}=\sum\left(a_{i}\left(\bar{Z}_{i}-\breve{Z}_{i}\right)+c_{i}\left|\breve{Z}_{i}\right|\right)=\sum a_{i} \bar{Z}_{i}-a_{i} \breve{Z}_{i}+c_{i}\left|\breve{Z}_{i}\right| \text {; }
$$

- the upper border of the interval

$$
\bar{y}=\sum\left(a_{i} \bar{Z}_{i}+c_{i} \mid \bar{Z}_{i}\right) \text {. }
$$

For the interval model to be correct, the real value of input variable Y should lay in the interval obtained by the method FGMDH.

So, the general requirements to a linear interval model are the following: to find such values of parameters $\left(\breve{A}_{i}, c_{i}\right)$ of fuzzy coefficients, which ensure $[6,8]$ :

1) observed values $y_{k}$ should locate in an estimation interval for $Y_{k}$ total width of the estimation interval should be minimal;

2 ) these requirements may be redefined as a task of linear programming $[6,8]$ :

$$
\left.\min _{a_{i}, c_{i}} \sum_{k=1}^{M}\left(\sum\left(a_{i} \bar{Z}_{i}+c_{i} \mid \breve{Z}_{i}\right)\right)-\sum\left(a_{i} \underline{Z}_{i}-c_{i}\left|\breve{Z}_{i}\right|\right)\right),
$$

under conditions

$$
\left\{\begin{array}{l}
\sum\left(a_{i} \underline{Z}_{i k}-c_{i}\left|\breve{Z}_{i k}\right|\right) \leq y_{k}, \\
\sum\left(a_{i} \bar{Z}_{k i}+c_{i}\left|\breve{Z}_{i k}\right|\right) \geq y_{k}, k=\overline{1, M .}
\end{array}\right.
$$

Let us consider partial description (1). Then math model takes the form [6]:

$$
\begin{gathered}
\min _{a_{i}, c_{i}}\left(2 M c_{0}+a_{1} \sum_{k=1}^{M}\left(\bar{x}_{i k}-\underline{x}_{i k}\right)+2 c_{1} \sum_{k=1}^{M}\left|\breve{x}_{i k}\right|+a_{2} \sum_{k=1}^{M}\left(\bar{x}_{j k}-\underline{x}_{j k}\right)+2 c_{2} \sum_{k=1}^{M}\left|\breve{x}_{j k}\right|+\right. \\
\left.+a_{3} \sum_{k=1}^{M}\left(\left|\breve{x}_{i k}\right|\left(\bar{x}_{j k}-\underline{x}_{j k}\right)+\left|\breve{x}_{j k}\right|\left(\bar{x}_{i k}-\underline{x}_{i k}\right)\right)+2 c_{3} \sum_{k=1}^{M}\left|\breve{x}_{i k} \breve{x}_{j k}\right|+2 a_{4} \sum_{k=1}^{M}\left|\breve{x}_{i k}\right| \bar{x}_{i k}-\underline{x}_{i k}\right)+ \\
\left.+2 c_{4} \sum_{k=1}^{M} \bar{x}_{i k}^{2}+2 a_{5} \sum_{k=1}^{M}\left|\breve{x}_{j k}\right|\left(\bar{x}_{j k}-\underline{x}_{j k}\right)+2 c_{5} \sum_{k=1}^{M} \bar{x}_{j k}^{2}\right),
\end{gathered}
$$

with the following constraints

$$
\begin{gathered}
a_{0}+a_{1} \underline{x}_{i k}+a_{2} \underline{x}_{j k}+a_{3}\left(-\left|\breve{x}_{i k}\right|\left(\breve{x}_{j k}-\underline{x}_{j k}\right)-\left|\breve{x}_{j k}\right|\left(\breve{x}_{i k}-\underline{x}_{i k}\right)+\breve{x}_{i k} \breve{x}_{j k}\right)+ \\
+a_{4}\left(-2\left|\breve{x}_{i k}\right|\left(\breve{x}_{i k}-\underline{x}_{i k}\right)+\breve{x}_{i k}^{2}\right)+a_{5}\left(2\left|\breve{x}_{j k}\right|\left(\breve{x}_{j k}-\underline{x}_{j k}\right)+\breve{x}_{j k}^{2}\right)- \\
\quad-c_{0}-c_{1}\left|\breve{x}_{i k}\right|-c_{2}\left|\breve{x}_{j k}\right|-c_{3}\left|\breve{x}_{i k} \breve{x}_{j k}\right|-c_{4} \breve{x}_{i k}^{2}-c_{5} \breve{x}_{j k}^{2} \leq y_{k} ; \\
a_{0}+a_{1} \bar{x}_{i k}+a_{2} \bar{x}_{j k}+a_{3}\left(\left|\breve{x}_{i k}\right|\left(\bar{x}_{j k}-\breve{x}_{j k}\right)+\left|\breve{x}_{j k}\right|\left(\bar{x}_{i k}-\breve{x}_{i k}\right)-\breve{x}_{i k} \breve{x}_{j k}\right)+ \\
+a_{4}\left(2\left|\breve{x}_{i k}\right|\left(\bar{x}_{i k}-\breve{x}_{i k}\right)-\breve{x}_{i k}^{2}\right)+a_{5}\left(2\left|\breve{x}_{j k}\right|\left(\bar{x}_{j k}-\breve{x}_{j k}\right)-\breve{x}_{j k}^{2}\right)+c_{0}+ \\
+c_{1}\left|\breve{x}_{i k}\right|+c_{2}\left|\breve{x}_{j k}\right|+c_{3}\left|\breve{x}_{i k} \breve{x}_{j k}\right|+c_{4} \breve{x}_{i k}^{2}+c_{5} \breve{x}_{j k}^{2} \geq y_{k}, \quad c_{l} \geq 0, \quad l=\overline{0,5} .
\end{gathered}
$$


As one can see, this is also the linear programming problem but there are still no constraints for non-negativity of variables $a_{i}$, so it is reasonable to pass to a dual problem introducing dual variables $\left\{\delta_{k}\right\}$ and $\left\{\delta_{k+M}\right\}$.

\section{Investigations of FGMDH with Fuzzy Inputs at Forecasting Problems}

The list of securities used for calculation of RTS index consists of the most liquid shares of Russian companies chosen by Information Committee and based on expert judgment. The number of securities may not exceed 50 .

The experiment contains 5 fuzzy input variables which are the stock prices of leading Russian energetic companies included into the list of RTS:

- index LKOH - shares of "Lukoil" joint-stock company;

- EESR - shares of "RAO UES of Russia" joint-stock company;

- YUKO - shares of "Yukos" joint-stock company;

- SNGSP - privileged shares of "Surgutneftegas" joint-stock company;

- SNGS - common shares of "Surgutneftegas" joint-stock company;

- Output variable is the value of RTS index (opening price) of the same pe$\operatorname{riod}(03.04 .2006-18.05 .2006)$.

The whole sample contains 32 instances (points) and training sample size is 18 points (optimal size of the training sample for current experiment). The results presented below were obtained in [8].

Experiment 1. For normalized input when using Gaussian MF in group method of data handling with fuzzy input data the results of experiment are presented (see Fig. 3) and table 5: for GMDH, MSE = 0,1129737, for FGMDH, $\mathrm{MSE}=0,0536556$.

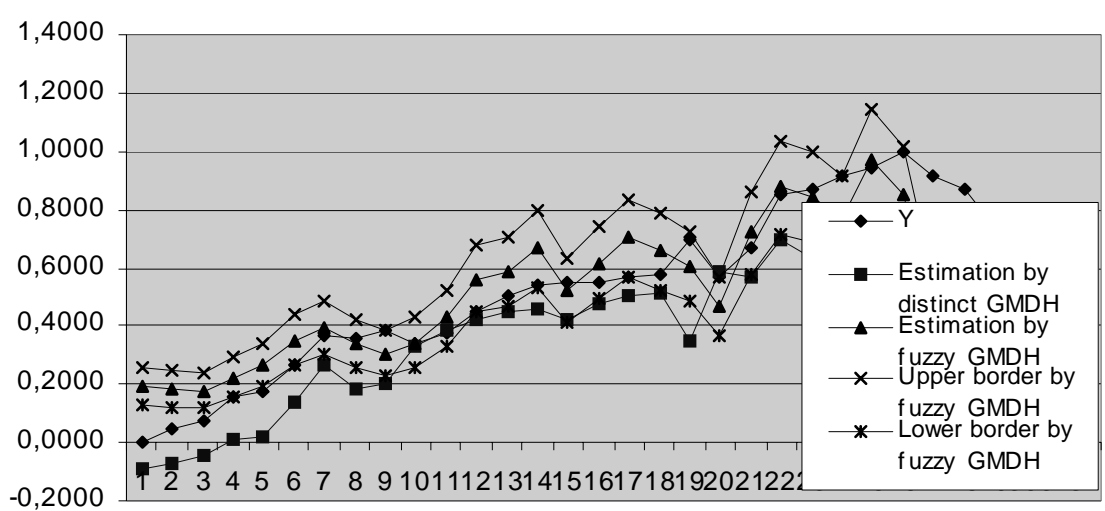

Fig. 3. Results of the Experiment1 using GMDH and FGMDH with fuzzy inputs

Table 5. MSE comparison for different methods at experiment 1

\begin{tabular}{|c|c|c|c|c|}
\hline Error & GMDH & FGMDH & $\begin{array}{c}\text { FGMDH with fuzzy } \\
\text { inputs, } \\
\text { Triangular MF }\end{array}$ & $\begin{array}{c}\text { FGMDH with fuzzy } \\
\text { inputs, } \\
\text { Gaussian MF }\end{array}$ \\
\hline MSE & 0,1129737 & 0,0536556 & 0,055557 & 0,028013 \\
\hline
\end{tabular}

As the results of experiment 1 show, fuzzy group method of data handling with fuzzy input data gives more accurate forecast than GMDH and FGMDH. In 
case of triangular MF FGMDH with fuzzy inputs gives a little worse forecast than FGMDH with Gaussian MF.

Experiment 2. RTS-2 index forecasting (opening price). The whole sample size is 32 instances and the training sample size is 18 ones. For normalized input data using Gaussian MF in FGMDH with fuzzy input data the following experimental results were obtained presented in the table 6 [8]: MSE for GMDH $=$ 0,051121, MSE for FGMDH $=0,063035$.

As the results of the experiment 2 show (Table 6), fuzzy group method of data handling with fuzzy input data gives better result than GMDH and FGMDH in case of both Gaussian and triangular membership functions.

Table 6. Comparison of different methods at experiment 2

\begin{tabular}{|c|c|c|c|c|}
\hline Error & GMDH & FGMDH & $\begin{array}{c}\text { FGMDH with fuzzy } \\
\text { inputs, } \\
\text { Triangular MF }\end{array}$ & $\begin{array}{c}\text { FGMDH with fuzzy } \\
\text { inputs, } \\
\text { Gaussian MF }\end{array}$ \\
\hline MSE & 0,051121 & 0,063035 & 0,061787 & 0,033097 \\
\hline
\end{tabular}

\section{CONCLUSION}

In this paper, the review of main results dealing with fuzzy inductive modeling method FGMDH is presented. This method enables to construct models of complex processes using experimental (statistical) data. Two different FGMDH versions were presented and discussed: with crisp inputs and fuzzy inputs.

The advantage of fuzzy GMDH is that it does not use least square method for search of unknown model coefficients opposite to classical GMDH and therefore the problem of possible ill-conditioned matrix does not exist for it.

Besides, fuzzy GMDH enables to find not point-wise forecast estimates but interval estimates for forecast values which allow to determine the forecast accuracy.

The generalization of fuzzy GMDH with fuzzy inputs was also considered and analyzed. The experimental investigations of GMDH and fuzzy GMDH in problems of share prices forecast at NYSE and Russian stock market RTS were carried out. The comparative results analysis has confirmed the high accuracy of fuzzy GMDH in problems of forecasting in financial sphere.

\section{REFERENCES}

1. Ivakhnenko A.G. Heuristic Self-Organization in Problems of Automatic Control / A.G. Ivakhnenko // Automatica (IFAC). - 1970. - 3. - P. 207-219.

2. Ivakhnenko A.G. Decision-making on the basis of self-organization / A.G. Ivakhnenko, Yu.P. Zaychenko, V.D. Dimitrov // Publ. house "Soviet Radio", Moscow, 1976.

3. Zaychenko Yu.P. Fuzzy group method of data handling and its application for macroeconomic indicators forecasting / Yu. P. Zaychenko, A.G. Kebkal, V.F. Krachkovsky // Scientific papers of NTUU "KPI". — 2000. — 2. — P. 18-26.

4. Zaychenko $Y u$. The investigations of different membership functions in Fuzzy Group Method of Data Handling / Yu. Zaychenko, I.O. Zayets, O.V. Kamotsky, O.V. Pavlyuk // Control Systems and machines. - 2003. - 2. - P. 56-67.

5. Zaychenko Yu.P. Synthesis and adaptation of fuzzy forecasting models on the basis of self-organization method / Yu.P. Zaychenko, I.O. Zayets // Scientific papers of NTUU “KPI”. — 2001. - 3. - P. 34-41. 
6. Zgurogvsky M. The fundamentals of computational intelligence: System approach / M. Zgurogvsky, Yu. Zaychenko // Springer. - Switzerland, 2016. - 308 p.

7. Zaychenko Yu. Fuzzy group method of data handling in forecasting problems at financial markets / Yu. Zaychenko // International Journal "Information Models and Analysis". - 2012. - 1(4). - P. 303-317.

8. Zaychenko Yu. The Investigations of Fuzzy Group Method of Data Handling with Fuzzy Inputs in the Problem of Forecasting in Financial Sphere / Yu. Zaychenko // Proceedings of the II International Conference on Inductive Modelling ICIM2008. — Kyiv: IRTC ITS NASU, 2008. — P. 129-133.

9. Huang $W$. Fuzzy polynomial neural networks: hybrid architectures of fuzzy modeling / W. Huang, S.K. Oh, W. Pedrycz // IEEE Transactions on Fuzzy Systems. 2002. - 10(5). - P. 607-621.

10. Oh S.K. Hybrid fuzzy polynomial neural networks / S.K. Oh, D.W. Kim, W. Pedrycz // International Journal of Uncertainty, Fuzziness and Knowledge-Based Systems. - 10(3). — https://doi.org/10.1142/S0218488502001478

11. Oh S.K. Self-Organizing Multi-layer Fuzzy Polynomial Neural Networks Based on Genetic Optimization / S.K. Oh, W. Pedrycz, B.J. Park // Fuzzy Sets and Systems. - 2004. - 145(1). - P.165-181.

12. Oh S.K. Self-organizing neuro-fuzzy networks in modeling software data / S.K. Oh, B.J. Park // Neurocomputing. - 2005. - 64. - P. 397-431.

13. Oh S.K. Design of Fuzzy Relation-Based Polynomial Neural Networks Using Information Granulation and Symbolic Gene Type Genetic Algorithms / S.K. Oh, W. Pedrycz, H.K. Kim // Advances in Neural Networks. Lecture Notes in Computer Science book series. — 2007. - Vol. 4492. - P. 206-215.

14. Oh S.K. Genetically optimized Hybrid Fuzzy Set-based Polynomial Neural Networks / S.K. Oh, W. Pedrycz, S.B. Roh // Journal of the Franklin Institute. - 2011. 348(2). - P. 415-425.

15. Oh S.K. Genetically Optimized Hybrid Fuzzy Neural Networks: Analysis and Design of Rule-based Multi-layer Perceptron Architectures / S.K. Oh, W. Pedrycz // Engineering Evolutionary Intelligent Systems. Part of the Studies in Computational Intelligence book series. - 2013. - Vol. 82. - P. 23-57.

16. Huang $W$. Hybrid fuzzy polynomial neural networks with the aid of weighted fuzzy clustering method and fuzzy polynomial neurons / W. Huang, S.K. Oh, W. Pedrycz. - Article. First Online: 15 September 2016.

17. Bodyanskiy Ye. Wavelet-neuro-fuzzy network structure optimization using GMDH for the solving forecasting tasks / Ye. Bodyanskiy, O. Vynokurova, A. Dolotov, O. Kharchenko // Proc. 4th Int. Conf. on Inductive Modeling. - Kyiv, 2013. P. 61-67.

18. Bodyanskiy Ye. Cascade GMDH-wavelet-neuro-fuzzy network / Ye. Bodyanskiy, O. Vynokurova, N. Teslenko // Proc. 4th Int. Workshop on Inductive Modeling. - Kyiv, 2011. - P. 22-30.

19. Bodyanskiy Ye. The neo-fuzzy neural network structure optimization using the GMDH for the solving forecasting and classification problems / Bodyanskiy Ye., Zaychenko Yu., Pavlikovskaya E. et al. // Proc. Int. Workshop on Inductive Modeling. - Krynica, Poland, 2009. - P. 77-89.

20. Bodyanskiy Ye.V. Self-learning cascade spiking neural network for fuzzy clustering based on Group Method of Data Handling / Ye.V. Bodyanskiy, O.A. Vynokurova, A.I. Dolotov // Journal of Automation and Information Sciences. 2013. - 45(3). - P. 23-33.

21. Zaychenko Yu. Hybrid GMDH-neuro-fuzzy system and its training scheme / Yu. Zaychenko, Ye. Bodyanskiy, O. Tyshchenko et al. // International Journal "Information Theory and Applications". - 2018. - 25(1). - P. 16-33.

Received 29.01.2019

From the Editorial Board: the article corresponds completely to submitted manuscript. 